\title{
Screening for alcohol use disorder among individuals with comorbid psychiatric disorders: Diagnostic accuracy in a sample of young Swiss men
}

\author{
Stéphanie Baggio ${ }^{\mathrm{a}, \mathrm{b}, \mathrm{c}, *}$, Sophie Baudat ${ }^{\mathrm{a}, \mathrm{d}}$, Jean-Bernard Daeppen ${ }^{\mathrm{e}}$, Gerhard Gmel $^{\mathrm{e}, \mathrm{f}, \mathrm{g}, \mathrm{h}}$, \\ Patrick Heller $^{\mathrm{a}, \mathrm{i}}$, Nader Perroud ${ }^{\mathrm{j}, \mathrm{k}, \mathrm{l}}$, Stéphane Rothen ${ }^{\mathrm{m}}$, Frank Sporkert ${ }^{\mathrm{n}}$, Joseph Studer ${ }^{\mathrm{e}}$, \\ Hans Wolff ${ }^{a}$, Katia Iglesias ${ }^{\circ}$ \\ ${ }^{a}$ Division of Prison Health, Geneva University Hospitals and University of Geneva, Chemin du Petit Bel Air 2, 1226 Thônex, Switzerland \\ ${ }^{\mathrm{b}}$ Department of Forensic Psychiatry, Institute of Forensic Medicine, University of Bern, Falkenplatz 16, 3012 Bern, Switzerland \\ ${ }^{\mathrm{c}}$ Office of Corrections, Zurich, Switzerland \\ ${ }^{\mathrm{d}}$ Family and Development Research Center, Institute of Psychology, University of Lausanne, Geopolis Building, 1015 Lausanne, Switzerland \\ ${ }^{\mathrm{e}}$ Addiction Medicine, Department of Psychiatry, Lausanne University Hospital, Rue du Bugnon 23, 1011 Lausanne, Switzerland \\ ${ }^{\mathrm{f}}$ Addiction Switzerland, Avenue Louis-Ruchonnet 14, 1003 Lausanne, Switzerland \\ ${ }^{\mathrm{g}}$ Centre for Addiction and Mental Health, 1001 Queen Street West, Toronto, ON M6J 1H4, Canada \\ ${ }^{\mathrm{h}}$ University of the West of England, Coldharbour Ln, Stoke Gifford, Bristol BS16 1QY, UK \\ ${ }^{\mathrm{i}}$ Adult Psychiatry Division, Department of Mental Health and Psychiatry, Geneva University Hospitals, Chemin du Petit Bel Air 2, 1226 Thônex, Switzerland \\ ${ }^{\mathrm{j}}$ Department of Mental Health and Psychiatry, Service of Psychiatric Specialties, University Hospitals of Geneva, Geneva, Switzerland \\ ${ }^{\mathrm{k}}$ Department of Psychiatry, University of Geneva, Geneva, Switzerland \\ ${ }^{1}$ Department of Psychiatry, Dalhousie University, Halifax, Nova Scotia, Canada \\ ${ }^{\mathrm{m}}$ Addiction Division, Geneva University Hospitals and University of Geneva, rue Grand-Pré 70C, 1202 Geneva, Switzerland \\ ${ }^{\mathrm{n}}$ Centre of Legal Medicine, Forensic Toxicology and Chemistry Unit, Lausanne and Geneva Universities, Chemin de la Vulliette 4, 1000 Lausanne, Switzerland \\ ${ }^{\circ}$ School of Health Sciences, HES-SO University of Applied Sciences and Arts of Western Switzerland, Route des Arsenaux 16a, 1700 Fribourg, Switzerland
}

\section{H I G H L I G H T S}

- High symptomatology on psychiatric disorders changed the optimal cut-off score of the AUD screening tool.

- AUD should be screening in conjunction with other psychiatric disorders.

- There is a need of an integrated approach to screening for AUD.

\section{A R T I C L E I N F O}

\section{Keywords:}

Alcohol

Comorbidity

Mental health

Population-based assessment

Psychometrics

Screening

\begin{abstract}
A B S T R A C T
Alcohol use disorder (AUD) is frequently comorbid with other psychiatric disorders. However, few studies investigated the psychometric properties of AUD screening tools in presence of co-occurring disorders. This study examined the diagnostic accuracy of a short AUD screening tool among young adults, in the presence of high $v s$. low or moderate symptomatology of other common psychiatric disorders. Data were collected among young Swiss men $(n=233)$ between 2016 and 2018. Measures included a diagnostic interview for AUD and screening tools for AUD and other psychiatric disorders (attention deficit hyperactivity disorder, antisocial personality disorder, bipolar disorder, borderline personality disorder, major depressive disorder, and social anxiety disorder). We computed receiver operating characteristic curves to test whether the AUD screening tool was an accurate indicator of AUD for groups with high $v$ s. low or moderate symptomatology of each psychiatric disorder. The results showed that the optimal cut-off score was $\geq 3$ (the original cut-off of the scale) for participants with a low or moderate symptomatology and $\geq 4$ for participants with a high symptomatology. Our findings highlighted the urgent need for an integrated approach to screening. Psychiatric comorbidities should be included in the screen for AUD to obtain accurate results.
\end{abstract}

\footnotetext{
* Corresponding author at: Chemin du Petit Bel Air 2, 1226 Thônex, Switzerland.

E-mail address: stephanie.baggio@hcuge.ch (S. Baggio).
} 


\section{Introduction}

Alcohol use disorder (AUD) is often comorbid with other psychiatric disorders. More than one third of AUD patients have another psychiatric disorder (Gimeno et al., 2017). Comorbid psychiatric disorders are usually associated with worse trajectories of both AUD and of the comorbid disorder: earlier onset, less response to treatment, more severe symptomatology, and higher relapse rates (Boden \& Moos, 2009; Gimeno et al., 2017; Pavkovic et al., 2018; Tolliver \& Anton, 2015). Integrated treatment taking into account comorbidities leads to better treatment outcomes (Kelly \& Daley, 2013). Therefore, identifying and treating potential comorbidities is essential (Priester et al., 2016). In this study, we focused on the critical first step of identifying AUD, which requires screening and assessment. Accurate screening tools are important for both general population-based assessments (e.g., public health planning) and in clinical settings. They are an efficient and costeffective method for preliminary diagnosis processes (Daigre et al., 2015).

Identifying AUD in populations with comorbid disorders is a complex process since symptoms of one disorder can distort the severity of the other disorder. Consequently, the accuracy of screening procedures is often compromised by a large rate of false positives for the established cut-off scores. As a consequence, individuals who do not have the disorder according to a reliable gold standard such as a diagnostic interview (e.g., ADHD with comorbid AUD: Luderer et al., 2019) are falsely identified as positive by the screening tool. To date, most studies investigated the psychometric properties of screening tools designed for various psychiatric disorders in presence of comorbid AUD (Delgadillo et al., 2011; Luderer et al., 2019), but not the psychometric properties of AUD screening tools in the presence of other psychiatric disorders. Comorbidity can also be associated with an overestimation of symptoms (e.g., AUD with major depressive disorder: Baggio et al., 2015), need of higher thresholds to provide an accurate diagnosis (for depression in presence of comorbid AUD or substance use disorder: Delgadillo et al., 2011) or overall poor psychometric performance (e.g., AUD with personality disorders: Kok, de Haan, Wieske, de Weert, \& de Jong, 2015). In addition, a recent study pointed out the lack of structured interview providing a reliable diagnosis of AUD, especially for the DSM-5 definition of AUD (Baggio \& Iglesias, 2019).

The lack of appropriate screening is especially salient on young adults. Young adulthood (18-30 years old) is a period in which substance use and related problems are the highest (Gmel et al., 2015). This is especially true for young men, who are heavier substance users than women. In addition, psychiatric disorders and substance use disorders are often related among young adults (Tretyak \& Welsh, 2019): Young people with psychiatric disorders have an increased risk of substance use disorders, and conversely, substance use disorders can lead to psychiatric problems.

This study investigated whether a short AUD screening tool (Baggio et al., 2019) accurately identified AUD among young men, taking into account symptomatology (high $v s$. low or moderate) on several common psychiatric disorders previously described as being associated with AUD: attention deficit hyperactivity disorder (ADHD, van Emmerik-van Oortmerssen et al., 2012), anxiety disorders (Gimeno et al., 2017), major depressive disorder (Pavkovic et al., 2018), bipolar disorder (Di Florio, Craddock, \& van den Bree, 2014), antisocial personality disorder (Compton, Conway, Stinson, Colliver, \& Grant, 2005), and borderline personality disorder (Trull et al., 2018).

\section{Methods}

\subsection{Participants and procedures}

Data were collected in the ongoing Cohort Study on Substance use and Risk Factors (C-SURF), which focuses on Swiss men aged 20-29 transitioning into adulthood (Gmel et al., 2015; Studer et al., 2013).
Participants were initially enrolled in 2010 during conscription in three Swiss national military recruitment centers. Participation in the C-SURF study was independent from the military recruitment. Among 6384 conscripts, 4430 (69.4\%) initially agreed to participate and signed an informed consent. Then, 5987 conscripts participated in the first wave of the study $(79.2 \%$ of those who gave written consent). The current study focused on the third wave, gathered average 61 months after the first wave. A total of 5445 participants filled out the questionnaire (retention rate from the first wave $=84.5 \%$ ).

A subsample of C-SURF participants was invited for a nested project, the Screening for Alcohol Dependence Among Young Swiss Men (SADYSM) study (Baggio et al., 2019; Iglesias, Sporkert, Daeppen, Gmel, \& Baggio, 2018). French-speaking young men who participated to the third wave of C-SURF and for whom valid email address was available $(n=2668)$ were invited to participate in the SADYSM study. They were first asked to complete the Alcohol Use Disorder Identification Test (AUDIT, Saunders, Aasland, Babor, Fuente, \& Grant, 1993) online (response rate $=51.4 \%$ ). Participants were then selected according to their AUDIT score to have a balanced number of participants with and without AUD (as diagnosed with the gold standard): those likely to have an AUD (AUDIT score $\geq 13$ ) and those not likely to have an AUD (AUDIT score < 13) (Meneses-Gaya et al., 2010). An appointment was scheduled at the Lausanne University Hospital for those who agreed to participate (response rate $=70.6 \%$ ). During the appointment, participants completed a self-reported computer-assisted questionnaire and underwent a structured interview designed to assess AUD with a trained psychologist.

In the present study, we used data of 233 participants involved in the third wave of C-SURF (2016-2017) and in the SADYSM study (2017-2018).

The Ethic Committee of the Canton of Vaud approved both studies (C-SURF: no. 15/07, SADYSM: no. 2017-00776).

\subsection{Measures}

AUD diagnosis and self-reported AUD were collected in the SADYSM study.

AUD diagnosis was assessed with the Diagnostic Interview for Genetic Studies (DIGS, Berney, Preisig, Matthey, Ferrero, \& Fenton, 2002) adapted for the DSM-5 (Baggio et al., 2019). The DIGS is a structured interview enabling a comprehensive and reliable assessment of AUD, with a high inter-rater agreement and a good concordance with clinical diagnoses (Berney et al., 2002). A cut-off score of 2 for the previous twelve months was used to define AUD in this study.

Self-reported AUD was assessed using a DSM-5 AUD screening tool. A recent study conducted in the same sample (Baggio et al., 2019) showed that this instrument, based on eight symptoms of AUD and four alcoholrelated consequences, was optimal to screen for AUD over the previous twelve months. The tool displays acceptable psychometric properties (sensitivity $=83.3 \%$, specificity $=78.7 \%$ ) with a cut-off of 3 . In this study, we used the sum score ranging from 0 to 12 to identify the optimal cut-off for different subgroups.

The severity of symptomatology of the following comorbid disorders was assessed using self-reported scales in the third wave of CSURF:

Attention deficit hyperactivity disorder (ADHD) was assessed with the six-item short version of the Adult ADHD Self Report Scale (Kessler et al., 2005). This scale has a good classification accuracy (97.9\%) and inter-rater reliability (Kappa $=0.76$ ) (Kessler et al., 2005). A total score of the items assessed on a five-point scale was computed, ranging from 6 to 30 (Cronbach alpha $=0.79$ ). It covered the previous twelve months.

Antisocial personality disorder was assessed using six items of the French version of the Mini International Neuropsychiatric Interview (Lecrubier et al., 1998), which have been described as a reliable unidimensional scale (Paap et al., 2017). Items are assessed on a six-point 
scale starting at the age of 20. A sum score ranging from 6 to 36 was computed (Cronbach alpha $=0.77$ ).

Bipolar disorder was measured using the Mood Disorder Questionnaire (Hirschfeld et al., 2000; Weber Rouget et al., 2005), which has been found to be reliable screening instrument (internal consistency: Cronbach alpha $=0.89$, test-retest: Kappa $=0.79$, Weber Rouget et al., 2005). A sum score ranging from 0 to 13 was computed based on the 13 true/false items (Cronbach alpha $=0.86$ ).

Borderline personality disorder was assessed using the Mclean Screening Instrument for Borderline Personality Disorder (Melartin, Häkkinen, Koivisto, Suominen, \& Isometsä, 2009; Zanarini et al., 2003). It includes ten true/false items with good psychometric properties (internal consistency: Cronbach alpha $=0.77$, Melartin et al., 2009; and good criterion validity, Patel, Sharp, \& Fonagy, 2011). We computed a sum score ranging from 0 to 10 (Cronbach alpha $=0.81$ ).

Major depressive disorder was assessed with the Major Depressive Inventory (Bech, Rasmussen, Olsen, Noerholm, \& Abildgaard, 2001). It consists of 12 six-point items assessed on a six-point scale, displaying good psychometric properties (internal consistency: Cronbach alpha $=0.90$, good content validity, Olsen, Jensen, Noerholm, Martiny, \& Bech, 2003). We computed a sum score ranging from 6 to 72 (Cronbach alpha $=0.92$ ). It covered the previous two weeks.

Social anxiety disorder was assessed with the Clinically Useful Social Anxiety Disorder Outcome Scale (Dalrymple et al., 2013), which has 12 items measured on a five-point scale. The scale has excellent psychometric properties (internal consistency: Cronbach alpha $=0.96$ and test-retest reliability: $r=0.89$, Dalrymple et al., 2013). We computed a sum score ranging from 0 to 48 (Cronbach alpha $=0.93$ ). It covered the previous week.

In addition to the sum scores that reflect the severity of each psychiatric disorder (a higher score indicated a higher severity), we created two groups for analytical purposes. They were defined as below or above the upper quartile (respectively: below: approx. $75 \%$ of observations and above: $25 \%$ of observations). These groups defined participants high $v$ s. low or moderate symptomatology. These groups were used because there were too few participants for most disorders when the recommended cut-off scores of the scales were used (ADHD: $15.9 \%, n=37$; antisocial personality disorder: $7.7 \%, n=18$; bipolar disorder: $8.2 \%, n=19$; borderline personality disorder: $9.0 \%, n=21$; major depressive disorder: $5.6 \%, n=13$; and social anxiety disorder: $20.6 \%, n=48$ ).

\subsection{Statistical analyses}

We first computed preliminary statistics for all variables, including percentages for binary variables and means for continuous and count variables. We tested the association between the severity (sum score) of each disorder and AUD diagnosis using negative binomial regressions.

We then used receiver operating characteristic (ROC) curves to test whether the AUD screening tool was an accurate indicator of AUD, using the diagnosis of the DIGS as the gold standard (Linnet, Bossuyt, Moons, \& Reitsma, 2012; Mandrekar, 2010). The ROC curve is a graphical representation of the diagnostic ability of a binary classifier (here, the AUD diagnosis) (Hajian-Tilaki, 2013). It plots the true positive rate (sensitivity, $x$-axis) against the false positive rate $(1-$ specificity, y-axis). We used parametric and non-parametric ROC curves, which address different research questions. We computed six parametric ROC regressions with maximum likelihood estimation using the sum scores for each psychiatric disorder. The parametric approach is used to obtain smooth ROC curves and to identify differences between curves. We also performed twelve non-parametric ROC curves separately for both groups (high $v s$. low or moderate symptomatology) for each psychiatric disorder. The non-parametric approach is used to obtain statistics for different cut-off scores and allows identification of the optimal cut-off score (high on sensitivity and low on 1 - specificity). For these non-parametric analyses, we derived sensitivity and specificity. We used the Youden's J statistic which gives an equal weight to both sensitivity and specificity (max(sensitivity + specificity) -1 ) to select the optimal cut-off score (Youden, 1950). All analyses were performed with Stata 15.

As sensitivity analyses, we tested whether the results were similar when we used groups defined as follows: 1 ) according to the clinical cut-off scores for the other psychiatric disorders, even if the sample size was low; and 2) as $60 \% / 40 \%$ instead of $75 \% / 25 \%$. Findings were similar to those reported in this study, except for major depression for the clinical cut-off score (optimal cut-off score $\geq 3$ in the group with high symptomatology). However, the group of participants meeting the threshold for major depression disorder was very small $(n=13)$ and thus this result should be interpreted very carefully.

\section{Results}

\subsection{Preliminary analyses}

Participants were on average $25.6 \pm 1.4$ years old when they completed the third wave of C-SURF and 27.0 when they participated in the SADYSM study. A total of $33.5 \%$ of the participants had a diagnosis of AUD. Sum scores for each scale assessing psychiatric disorders are reported in Table 1. Overall, participants with an AUD diagnosis scored significantly higher on all disorders. Descriptive statistics for groups according to the upper quartile for each psychiatric disorder are reported in Table 2 .

\subsection{ROC regressions and ROC curves}

All psychiatric disorders showed a statistically significant effect on the ROC curve for either the sum score or the group analysis (Table 3). Results were statistically significant for both measures for antisocial personality disorder $(\mathrm{p}=.003$ and $\mathrm{p}<.001)$, borderline personality disorder $(\mathrm{p}=.016$ and $\mathrm{p}=.022)$, and major depressive disorder

Table 1

Severity (sum scores) of psychiatric disorders.

\begin{tabular}{|c|c|c|c|c|c|c|c|}
\hline \multirow[b]{2}{*}{ Psychiatric disorders } & \multicolumn{2}{|l|}{ Overall } & \multicolumn{2}{|c|}{ non-AUD group $(n=155)$} & \multicolumn{2}{|c|}{ AUD group $(n=78)$} & \multirow[t]{2}{*}{ Severity $^{1}$} \\
\hline & Mean (sd) & Median & Mean (sd) & Median & Mean (sd) & Median & \\
\hline AUD screening tool $(0-12)$ & $2.6(2.2)$ & 2 & $1.7(1.5)$ & 2 & $4.5(2.1)$ & 4 & $<0.001$ \\
\hline Antisocial personality disorder $(6-30)$ & $9.1(3.9)$ & 8 & $8.4(3.5)$ & 8 & $10.5(4.4)$ & 9 & $<0.001$ \\
\hline Attention deficit hyperactivity disorder (6-36) & $14.3(4.6)$ & 15 & $13.7(4.2)$ & 14 & $15.6(5.1)$ & 16 & 0.002 \\
\hline Bipolar disorder $(0-12)$ & $4.6(3.7)$ & 4 & $3.9(3.4)$ & 3 & $5.8(3.8)$ & 5 & 0.001 \\
\hline Borderline personality disorder (0-9) & $2.5(2.6)$ & 2 & $2.0(2.2)$ & 1 & $3.7(2.9)$ & 3 & $<0.001$ \\
\hline Major depressive disorder (6-72) & $10.8(8.5)$ & 8 & $9.3(7.0)$ & 7 & $14.0(10.1)$ & 10 & $<0.001$ \\
\hline Social anxiety disorder $(0-48)$ & $8.4(8.8)$ & 6 & $7.2(8.3)$ & 4 & $10.8(9.4)$ & 9 & 0.017 \\
\hline
\end{tabular}

AUD: Alcohol use disorder, sd: standard deviation.

1 Negative binomial regression (predictor: AUD diagnosis, outcome: sum score of each psychiatric disorder). 
Table 2

Groups defined according to the upper quartile for psychiatric disorders.

\begin{tabular}{lll}
\hline Psychiatric disorder & $\%(\mathrm{n})$ & Mean (sd) \\
\hline Attention deficit/hyperactivity disorder & & \\
$\quad$ Score $<17$ & $70.4(164)$ & $12.1(3.2)$ \\
$\quad$ Score $\geq 17$ & $29.6(69)$ & $19.6(2.7)$ \\
Antisocial personality disorder & & \\
$\quad$ Score $<11$ & $74.7(174)$ & $7.3(1.2)$ \\
$\quad$ Score $\geq 11$ & $25.3(59)$ & $14.4(4.3)$ \\
Bipolar disorder & & \\
$\quad$ Score $<7$ & $69.5(162)$ & $2.5(2.0)$ \\
$\quad$ Score $\geq 7$ & $30.5(71)$ & $9.2(1.9)$ \\
Borderline personality disorder & & \\
$\quad$ Score $<4$ & $69.1(161)$ & $1.1(1.2)$ \\
$\quad$ Score $\geq 4$ & $30.9(72)$ & $5.8(1.7)$ \\
Major depressive disorder & & \\
$\quad$ Score $<14$ & $74.1(172)$ & $6.7(3.1)$ \\
$\quad$ Score $\geq 14$ & $25.9(60)$ & $22.7(7.6)$ \\
Social anxiety disorder & & \\
$\quad$ Score $<13$ & $73.4(171)$ & $3.9(3.9)$ \\
$\quad$ Score $\geq 13$ & $26.6(62)$ & $20.7(6.7)$
\end{tabular}

sd: standard deviation.

( $\mathrm{p}<.001)$; for sum score for social anxiety disorder $(\mathrm{p}=.031)$; and for group analysis for ADHD $(\mathrm{p}=.050)$ and bipolar disorder $(\mathrm{p}=.038)$. Results for each disorder are presented by group (high $v s$. low or moderate symptomatology) in Fig. 1. In nearly all cases, the presence of high symptomatology appeared to increase the diagnostic accuracy of the AUD screening tool to detect AUD.

The optimal cut-off scores of the AUD screening tool for each psychiatric disorder are reported in Table 3. The optimal cut-off scores differed between groups. For participants with low or moderate symptomatology, the optimal cut-off score was systematically $\geq 3$, which corresponds to the original cut-off of the scale. For participants with a high symptomatology, the optimal AUD cut-off score was $\geq 4$. When a cut-off $\geq 3$ was used for the latter, specificity was lower for all disorders: ADHD (79.0\% for the cut-off of $3 v s .92 .1 \%$ for the cut-off of 4), antisocial personality disorder ( $57.8 \% v s .76 .9 \%)$, bipolar disorder (62.5\% vs. $77.5 \%)$, borderline personality disorder (63.2\% vs. $79.0 \%)$, major depressive disorder $(71.0 \%$ vs. $80.7 \%)$, and social anxiety disorder $(56.7 \%$ vs. $70.0 \%)$. On the contrary, a cut-off score of 4 among participants with a low or moderate symptomatology yielded lower sensitivities: ADHD (68.1\% for the cut-off of $4 v s .83 .0 \%$ for the cut-off of 3), antisocial personality disorder $(64.4 \%$ vs. $73.3 \%)$, bipolar disorder $(72.3 \%$ vs. $83.0 \%)$, borderline personality disorder $(70.5 \%$ vs. $81.8 \%)$, major depressive disorder $(60.4 \%$ vs. $75.0 \%)$, and social anxiety disorder $(71.7 \%$ vs. $80.4 \%)$.

Interestingly, participants with high symptomatology on psychiatric disorders other than AUD (cut-off $\geq 4$ ) were better classified than those with low or moderate symptomatology (cut-off $\geq 3$ ) for most disorders (higher Youden's J statistics for ADHD, antisocial personality disorder, borderline personality disorder, and major depression disorder).

\section{Discussion}

\subsection{Main findings}

This study tested the diagnostic accuracy of an AUD screening tool among participants with varying symptomatology on other common psychiatric disorders: ADHD, antisocial personality disorder, bipolar disorder, borderline personality disorder, major depression disorder, and social anxiety disorder. The results showed that the symptomatology of all of these psychiatric disorders influenced the results/outcome of the AUD screening tool, using both severity (sum scores of selfreported scales for the other psychiatric disorders) and a binary classification of participants having or not a high level of symptomatology on the other psychiatric disorder (within the upper quartile of the distribution of sum scores). Importantly, the optimal cut-off score changed according to the severity of the symptomatology of the other psychiatric disorders: for participants with a high level of symptomatology, the cut-off score of 3 recommended for the general population by the AUD screening tool (Baggio et al., 2019) should be increased to 4. Otherwise, the rate of false positives was high, ranging from $21.0 \%$ to $43.3 \%$. On the contrary, the rate of false positives was lower with a threshold of $4(7.9-30.0 \%)$. This result is in line with other studies reporting a reduced sensitivity for screening tools when comorbid disorders are present (Kok et al., 2015; Luderer et al., 2019; Weibel et al., 2018) or the need of higher thresholds for screening tools used in population having comorbid disorders (Delgadillo et al., 2011).

Overall, using the AUD screening tool with the recommended threshold ( $\geq 3$ in this case) would lead to an overestimation of AUD in presence of a high symptomatology on other psychiatric disorders. This finding was in line with a previous study reporting that depression might worsen the evaluation of AUD symptoms, independently of

Table 3

Psychometric properties for ROC curves according to severity/symptomatology of psychiatric disorders: Differences based on AUD cut-off scores.

\begin{tabular}{|c|c|c|c|c|c|c|c|c|}
\hline \multirow[t]{2}{*}{ Psychiatric disorder } & \multicolumn{2}{|l|}{$\mathrm{n}$} & \multicolumn{2}{|c|}{$\mathrm{p}$-value ROC regressions } & \multicolumn{2}{|c|}{ Recommended cut-off $(\geq 3)$} & \multicolumn{2}{|c|}{ Alternative cut-off ( $\geq 4)$} \\
\hline & non-AUD group & AUD group & Sum score & Groups & Sensitivity & Specificity & Sensitivity & Specificity \\
\hline \multicolumn{9}{|l|}{ Attention deficit/hyperactivity disorder } \\
\hline Low or moderate symptomatology $($ score $<17$ ) & 17 & 47 & 0.055 & 0.050 & 83.0 & 78.6 & 68.1 & 86.3 \\
\hline High symptomatology (score $\geq 17$ ) & 38 & 31 & & & 83.9 & 79.0 & 80.7 & 92.1 \\
\hline \multicolumn{9}{|l|}{ Antisocial personality disorder } \\
\hline Low or moderate symptomatology (score $<11$ ) & 129 & 45 & 0.003 & $<0.001$ & 73.3 & 83.0 & 64.4 & 89.9 \\
\hline High symptomatology (score $\geq 11$ ) & 26 & 33 & & & 97.0 & 57.8 & 84.9 & 76.9 \\
\hline \multicolumn{9}{|l|}{ Bipolar disorder } \\
\hline Low or moderate symptomatology (score $<7$ ) & 115 & 47 & 0.089 & 0.038 & 83.0 & 84.4 & 72.3 & 91.3 \\
\hline High symptomatology (score $\geq 7$ ) & 40 & 31 & & & 83.9 & 62.5 & 74.2 & 77.5 \\
\hline \multicolumn{9}{|l|}{ Borderline personality disorder } \\
\hline Low or moderate symptomatology $($ score $<4$ ) & 17 & 44 & 0.016 & 0.022 & 81.8 & 83.8 & 70.5 & 90.6 \\
\hline High symptomatology (score $\geq 4$ ) & 38 & 34 & & & 85.3 & 63.2 & 76.5 & 79.0 \\
\hline \multicolumn{9}{|l|}{ Major depressive disorder } \\
\hline Low or moderate symptomatology $($ score $<14$ ) & 124 & 48 & $<0.001$ & $<0.001$ & 75.0 & 80.7 & 60.4 & 89.5 \\
\hline High symptomatology (score $\geq 14$ ) & 31 & 29 & & & 100 & 71.0 & 96.6 & 80.7 \\
\hline \multicolumn{9}{|l|}{ Social anxiety disorder } \\
\hline Low or moderate symptomatology (score $<13$ ) & 125 & 46 & 0.031 & 0.085 & 80.4 & 84.0 & 71.7 & 92.0 \\
\hline High symptomatology (core $\geq 13$ ) & 30 & 32 & & & 87.5 & 56.7 & 75.0 & 70.0 \\
\hline
\end{tabular}

AUD: Alcohol use disorder, ROC: receiver operating characteristics, CI: confidence interval.

The optimal AUD cut-off scores are highlighted in bold. 

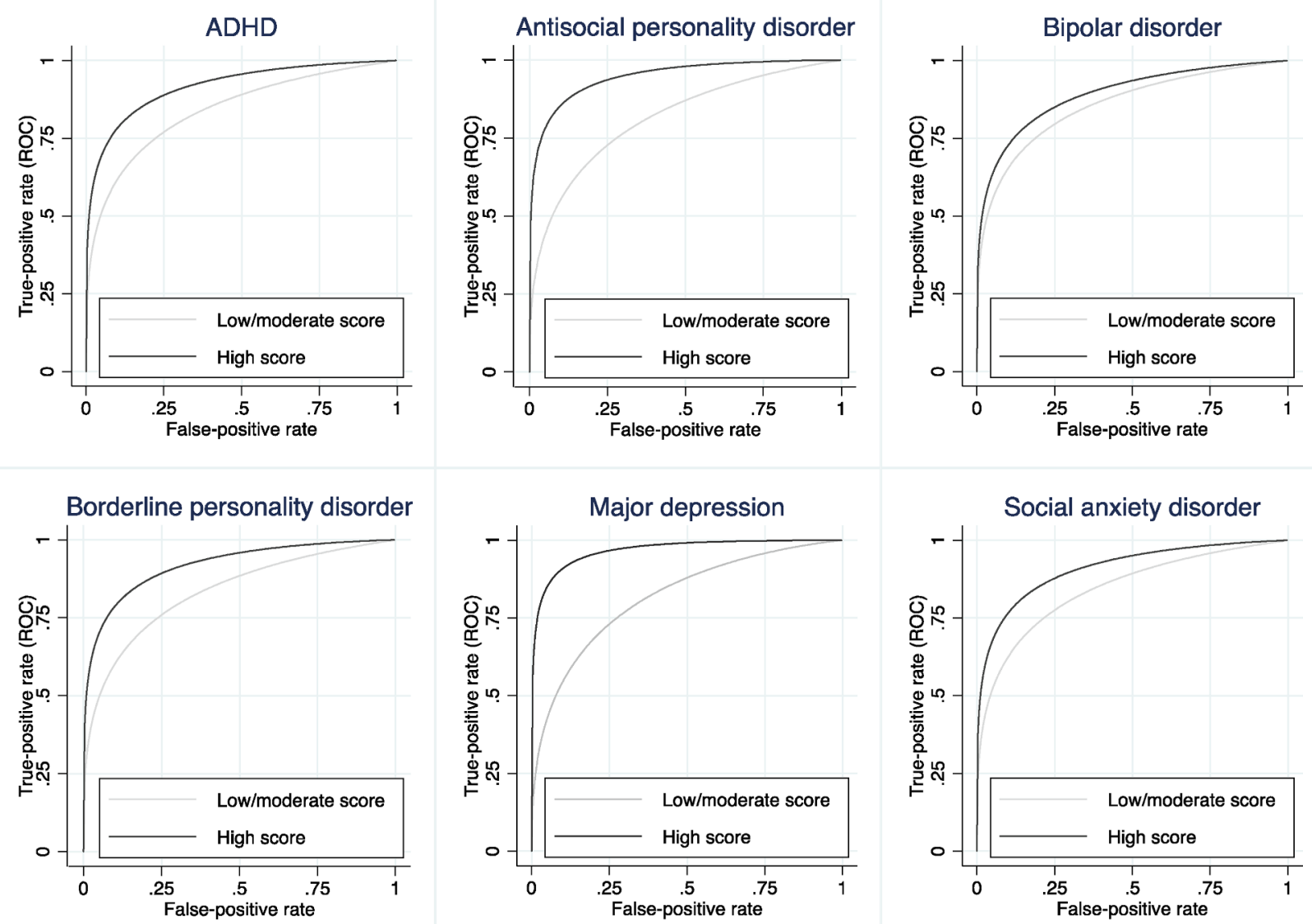

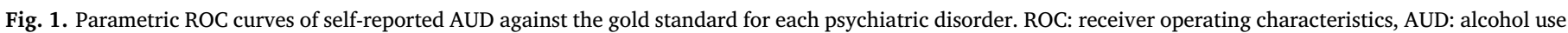

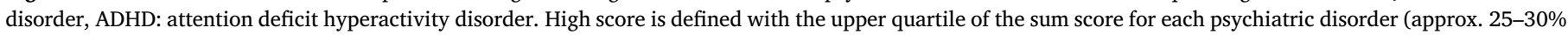
of participants with the highest scores).

alcohol use (Baggio et al., 2015). As a consequence, too many individuals might be referred to a full AUD diagnostic interview, increasing health care costs. Other consequences include the potential for increased emotional burden on individuals who screen positive but do not have AUD and need to undergo further assessments. Other undesirable consequences are risks of increased stigma and lack of reliable estimates of prevalence rates for public health purposes and planning of prevention and early intervention.

Ideally, a substance use screening tool should provide a consistent standardized measure of the disorder (Scheffler, 2013). Our study showed that standardizing the cut-off score of self-reported AUD for the general population was difficult (Baggio \& Iglesias, 2019). Even worse, it seems that it is impossible to screen for AUD without considering other psychiatric disorders that may inflate its prevalence rate. The threshold should be altered in certain subgroups to improve diagnostic accuracy of screening tools. Our findings highlighted the crucial need of an integrated approach to screening, as it has already been recommended for treatment (Scheffler, 2013). It means that other psychiatric disorders should be taken into account when screening for AUD, since simultaneous screening allows to determine the optimal cutoff score. This integrated perspective would be relatively easy to implement in clinical settings. Our conclusions also have important implications for public health and screening, as an integrated approach would require longer questionnaires. Even if it is costly and time-consuming, this may be the best way to promote a more holistic and accurate treatment approach.

\subsection{Limitations}

This study has some shortcomings. First, the sample might not be representative for the whole Swiss population of young men, as some of them declined to participate in the study (Studer et al., 2013). Second, it used self-reported scales to assess psychiatric disorders other than AUD. Self-reported scales are sometimes not accurate, especially in the presence of AUD. For example, ADHD symptoms are likely to be underreported among alcohol-dependent patients (Luderer et al., 2019). Third, we relied on a sample of young Swiss males, who constitute a specific group of alcohol drinkers. A fourth shortcoming was that psychiatric disorders were assessed in the third wave of C-SURF, on average 17 months before the SADYSM study started. The symptomatology of psychiatric disorders might have changed between the two time points. Thus, replication studies among females, other age groups, clinical populations, larger samples, and studies using diagnostic interviews along with concurrent assessment are needed to confirm our findings. Another limitation was the modest sample size, which might have led to marginal instead of significant effects (parametric ROC curves for ADHD, bipolar disorder, and social anxiety disorder). Furthermore, we compared "high" vs. "low or moderate" scoring groups on psychiatric disorders because the sample size was too small to use the recommended thresholds. Consequently, our results should be interpreted in line with this important limitation. Finally, our study did not take into account multiple comorbidities and how some symptoms might interfere with AUD screening outcomes more strongly than others, so future research should investigate these important questions. 


\subsection{Conclusion}

Researchers and clinicians should be aware that cut-off scores need to be adapted according to other characteristics of the population, namely the symptomatology on other psychiatric disorders. For research purposes and whenever possible in clinical settings, we suggest to consider disorders as constructs underpinning severity (i.e., relying on sum scores or number of symptoms) instead of using binary classifications (Kerridge, Saha, Gmel, \& Rehm, 2013; Liu, 2017). More generally, screening tools should be used in an integrated approach, meaning that psychiatric disorders should be considered as a whole and not as separate independent entities.

\section{Contributions}

The study's objectives were developed by StB and KI. StB and KI collected data of the SADYSM study. GG and JS developed the C-SURF study and collected its data. StB drafted the manuscript and performed statistical analyses. All co-authors made substantial contributions to the conception of the study and its design and made substantial contributions for data interpretation. All authors critically reviewed the manuscript for important intellectual content. All authors read and approved the final manuscript.

\section{Funding}

The Swiss National Science Foundation funded both studies used in this manuscript (no. 33CS30-148493 and no. 10001C_173418/1).

\section{Declaration of Competing Interest}

The authors declare that they have no known competing financial interests or personal relationships that could have appeared to influence the work reported in this paper.

\title{
Appendix 1. Alcohol use disorder screening tool
}

\author{
In the past 12 months... \\ Did you more than once drive a car or another vehicle (such a car, bicycle, motorcycle or moped) shortly after you had had several dinks with alcohol? \\ after you had been drinking too much alcohol? \\ Did you find you needed a lot more alcohol to become high o drunk than you used to? \\ Did you start feeling nervous or shaky for a full day or more after you had cut down on your drinking? \\ Did you try to cut down on your drinking, but couldn't? \\ Did you find yourself spending a great deal of time obtaining, using, or recovering from the effects of alcohol? \\ Did you give up activities you care about (e.g., school, work or being with friends and family) because of your drinking? \\ Did you continue drinking even though you were aware that alcohol had repeatedly caused you anxiety, depression or health problems? \\ Have you had such a strong desire or urge to drink that you could not help drinking? \\ In the last 12 months, it happened that... \\ While drinking alcohol, I did something that I badly regretted later. \\ I had unplanned sex because I was drunk \\ I had an accident or I got injured because I was drunk. \\ I came into a conflict with the police or with the authorities more than once because of my alcohol use.
}

Did you find yourself more than once in a situation that increased your chances of getting injured (sing machines, walking or doing sport in a dangerous area or around heavy traffic)

\section{Appendix A. Supplementary data}

Supplementary data to this article can be found online at https://doi.org/10.1016/j.addbeh.2020.106354.

\section{References}

Baggio, S., \& Iglesias, K. (2019). Commentary on Campbell and Strickland (2019): Caution is needed when using self-reported alcohol use disorder screening tools. Addictive Behaviors 106115.

Baggio, S., Iglesias, K., Studer, J., Dupuis, M., Daeppen, J.-B., \& Gmel, G. (2015). Is the relationship between major depressive disorder and self-reported alcohol use disorder an artificial one? Alcohol and Alcoholism (Oxford, Oxfordshire), 50(2), 195-199.

Baggio, S., Trächsel, B., Rousson, V., Rothen, S., Studer, J., Marmet, S., ... Iglesias, K. (2019). Identifying an accurate self-reported screening tool for alcohol use disorder: Evidence from a population-based assessment. Addiction in press.

Bech, P., Rasmussen, N. A., Olsen, L. R., Noerholm, V., \& Abildgaard, W. (2001). The sensitivity and specificity of the Major Depression Inventory, using the Present State Examination as the index of diagnostic validity. Journal of Affective Disorders, 66(2), 159-164.

Berney, A., Preisig, M., Matthey, M.-L., Ferrero, F., \& Fenton, B. T. (2002). Diagnostic interview for genetic studies (DIGS): Inter-rater and test-retest reliability of alcohol and drug diagnoses. Drug and Alcohol Dependence, 65(2), 149-158.

Boden, M. T., \& Moos, R. (2009). Dually diagnosed patients' responses to substance use disorder treatment. Journal of Substance Abuse Treatment, 37(4), 335-345.

Compton, W. M., Conway, K. P., Stinson, F. S., Colliver, J. D., \& Grant, B. F. (2005). Prevalence, correlates, and comorbidity of DSM-IV antisocial personality syndromes and alcohol and specific drug use disorders in the United States: Results from the national epidemiologic survey on alcohol and related conditions. The Journal of Clinical Psychiatry, 66(6), 677-685.

Daigre, C., Roncero, C., Rodríguez-Cintas, L., Ortega, L., Lligoña, A., Fuentes, S., ... Casas, M. (2015). Adult ADHD screening in alcohol-dependent patients using the WenderUtah rating scale and the adult ADHD self-report scale. Journal of Attention Disorders, 19(4), 328-334.

Dalrymple, K., Martinez, J., Tepe, E., Young, D., Chelminski, I., Morgan, T., \& Zimmerman, M. (2013). A clinically useful social anxiety disorder outcome scale.
Comprehensive Psychiatry, 54(7), 758-765.

Delgadillo, J., Payne, S., Gilbody, S., Godfrey, C., Gore, S., Jessop, D., \& Dale, V. (2011) How reliable is depression screening in alcohol and drug users? A validation of brief and ultra-brief questionnaires. Journal of Affective Disorders, 134(1), 266-271.

Di Florio, A., Craddock, N., \& van den Bree, M. (2014). Alcohol misuse in bipolar disorder. A systematic review and meta-analysis of comorbidity rates. European Psychiatry: The Journal of the Association of European Psychiatrists, 29(3), 117-124.

Gimeno, C., Dorado, M. L., Roncero, C., Szerman, N., Vega, P., Balanzá-Martínez, V., \& Alvarez, F. J. (2017). Treatment of comorbid alcohol dependence and anxiety disorder: Review of the scientific evidence and recommendations for treatment. Frontiers in Psychiatry, 8, 173.

Gmel, G., Akre, C., Astudillo, M., Bähler, C., Baggio, S., Bertholet, N., ... Wang, J. (2015). The Swiss Cohort Study on substance use risk factors - Findings of two waves. SUCHT, 61(4), 251-262.

Hajian-Tilaki, K. (2013). Receiver operating characteristic (ROC) curve analysis for medical diagnostic test evaluation. Caspian Journal of Internal Medicine, 4(2), 627-635.

Hirschfeld, R. M. A., Williams, J. B. W., Spitzer, R. L., Calabrese, J. R., Flynn, L., Keck, P. E., ... Zajecka, J. (2000). Development and validation of a screening instrument for bipolar spectrum disorder: the mood disorder questionnaire. American Journal of Psychiatry, 157(11), 1873-1875.

Iglesias, K., Sporkert, F., Daeppen, J.-B., Gmel, G., \& Baggio, S. (2018). Comparison of self-reported measures of alcohol-related dependence among young Swiss men: A study protocol for a cross-sectional controlled sample. BMJ Open, 8(7), e023632.

Kelly, T. M., \& Daley, D. C. (2013). Integrated treatment of substance use and psychiatric disorders. Social Work in Public Health, 28(3-4), 388-406.

Kerridge, B. T., Saha, T. D., Gmel, G., \& Rehm, J. (2013). Taxometric analysis of DSM-IV and DSM-5 alcohol use disorders. Drug and Alcohol Dependence, 129(1-2), 60-69.

Kessler, R. C., Adler, L., Ames, M., Demler, O., Faraone, S., Hiripi, E., ... Walters, E. E. (2005). The World Health Organization adult ADHD self-report scale (ASRS): A short screening scale for use in the general population. Psychological Medicine, 35(2), 245-256. 
Kok, T., de Haan, H., Wieske, E., de Weert, G., \& de Jong, C. (2015). Screening for personality disorders in outpatient substance use disorder patients. European Journal of Psychological Assessment, 33(3), 166-172.

Lecrubier, Y., Weiller, E., Hergueta, T., Amorim, P., Bonora, L. I., \& Lépine, J. P. (1998). MINI Mini International Neuropsychiatric Interview. French version 5.0.0. Paris. France: Hôpital de la Pitié Salpétrière, INSERM.

Linnet, K., Bossuyt, P. M. M., Moons, K. G. M., \& Reitsma, J. B. (2012). Quantifying the accuracy of a diagnostic test or marker. Clinical Chemistry, 58(9), 1292-1301.

Liu, R. T. (2017). Substance use disorders in adolescence exist along continua: Taxometric evidence in an epidemiological sample. Journal of Abnormal Child Psychology, 45(8), 1577-1586.

Luderer, M., Kaplan-Wickel, N., Richter, A., Reinhard, I., Kiefer, F., \& Weber, T. (2019). Screening for adult attention-deficit/hyperactivity disorder in alcohol dependent patients: Underreporting of ADHD symptoms in self-report scales. Drug and Alcohol Dependence, 195, 52-58.

Mandrekar, J. N. (2010). Simple statistical measures for diagnostic accuracy assessment. Journal of Thoracic Oncology, 5(6), 763-764.

Melartin, T., Häkkinen, M., Koivisto, M., Suominen, K., \& Isometsä, E. (2009). Screening of psychiatric outpatients for borderline personality disorder with the McLean Screening Instrument for Borderline Personality Disorder (MSI-BPD). Nordic Journal of Psychiatry, 63(6), 475-479.

Meneses-Gaya, C., Zuardi, A. W., Loureiro, S. R., Hallak, J. E. C., Trzesniak, C., de Azevedo Marques, J. M., ... Crippa, J. A. S. (2010). Is the full version of the AUDIT really necessary? Study of the validity and internal construct of its abbreviated versions. Alcoholism, Clinical and Experimental Research, 34(8), 1417-1424.

Olsen, L. R., Jensen, D. V., Noerholm, V., Martiny, K., \& Bech, P. (2003). The internal and external validity of the Major Depression Inventory in measuring severity of depressive states. Psychological Medicine, 33(2), 351-356.

Paap, M. C. S., Braeken, J., Pedersen, G., Urnes, Ø., Karterud, S., Wilberg, T., \& Hummelen, B. (2017). A psychometric evaluation of the DSM-IV criteria for antisocial personality disorder: dimensionality, local reliability, and differential item functioning across gender. Assessment 1073191117745126.

Patel, A. B., Sharp, C., \& Fonagy, P. (2011). Criterion validity of the MSI-BPD in a community sample of women. Journal of Psychopathology and Behavioral Assessment, 33(3), 403-408.

Pavkovic, B., Zaric, M., Markovic, M., Klacar, M., Huljic, A., \& Caricic, A. (2018). Double screening for dual disorder, alcoholism and depression. Psychiatry Research, 270, 483-489.
Priester, M. A., Browne, T., Iachini, A., Clone, S., DeHart, D., \& Seay, K. D. (2016). Treatment access barriers and disparities among individuals with co-occurring mental health and substance use disorders: An integrative literature review. Journal of Substance Abuse Treatment, 61, 47-59.

Saunders, J. B., Aasland, O. G., Babor, T. F., Fuente, J. R. D. L., \& Grant, M. (1993) Development of the Alcohol Use Disorders Identification Test (AUDIT): WHO collaborative Project on early detection of persons with harmful alcohol consumption-II. Addiction, 88(6), 791-804.

Scheffler, S. (2013). Assessment and treatment of clients with co-occurring psychiatric and substance use disorders. Clinical work with substance-abusing clients, third edition (pp. 371-395). New York, NY, US: Guilford Press.

Studer, J., Baggio, S., Mohler-Kuo, M., Dermota, P., Gaume, J., Bertholet, N., ... Gmel, G. (2013). Examining non-response bias in substance use research-are late respondents proxies for non-respondents? Drug and Alcohol Dependence, 132(1-2), 316-323.

Tolliver, B. K., \& Anton, R. F. (2015). Assessment and treatment of mood disorders in the context of substance abuse. Dialogues in Clinical Neuroscience, 17(2), 181-190.

Tretyak, V., \& Welsh, J. W. (2019). Co-occurring mental health disorders. In J. W. Welsh, \& S. E. Hadland (Eds.). Treating adolescent substance use: A clinician's guide (pp. 55-65). Cham: Springer International Publishing.

Trull, T. J., Freeman, L. K., Vebares, T. J., Choate, A. M., Helle, A. C., \& Wycoff, A. M. (2018). Borderline personality disorder and substance use disorders: An updated review. Borderline Personality Disorder and Emotion Dysregulation, 5(1), 15.

van Emmerik-van Oortmerssen, K., van de Glind, G., van den Brink, W., Smit, F., Crunelle, C. L., Swets, M., \& Schoevers, R. A. (2012). Prevalence of attention-deficit hyperactivity disorder in substance use disorder patients: A meta-analysis and meta-regression analysis. Drug and Alcohol Dependence, 122(1), 11-19.

Weber Rouget, B., Gervasoni, N., Dubuis, V., Gex-Fabry, M., Bondolfi, G., \& Aubry, J.-M. (2005). Screening for bipolar disorders using a French version of the Mood Disorder Questionnaire (MDQ). Journal of Affective Disorders, 88(1), 103-108.

Weibel, S., Nicastro, R., Prada, P., Cole, P., Rüfenacht, E., Pham, E., ... Perroud, N. (2018) Screening for attention-deficit/hyperactivity disorder in borderline personality disorder. Journal of Affective Disorders, 226, 85-91.

Youden, W. J. (1950). Index for rating diagnostic tests. Cancer, 3(1), 32-35.

Zanarini, M. C., Vujanovic, A. A., Parachini, E. A., Boulanger, J. L., Frankenburg, F. R., \& Hennen, J. (2003). A screening measure for BPD: The McLean Screening Instrument for Borderline Personality Disorder (MSI-BPD). Journal of Personality Disorders, 17(6), $568-573$. 